\title{
Nomogram to Predict Successful Septal Collateral Crossing during Percutaneous Coronary Intervention of Chronic Total Occlusion by retrograde approach: The Sep-CTO score
}

Tong Liu ( $\square$ liutong03@126.com )

Beijing Anzhen Hospital

Yuchao Zhan

Beijing Anzhen Hospital

Zheng Wu

Beijing Anzhen Hospital

Yun Lv

Beijing Anzhen Hospital

Wenzheng Li

Beijing Anzhen Hospital

Jinghua Liu

Beijing Anzhen Hospital

\section{Research Article}

Keywords: chronic total occlusion, percutaneous coronary intervention, septal collateral, nomogram, SepCTO score

Posted Date: April 9th, 2021

DOI: https://doi.org/10.21203/rs.3.rs-386483/v1

License: (c) (i) This work is licensed under a Creative Commons Attribution 4.0 International License. Read Full License 


\section{Abstract}

Septal collaterals are the main collaterals used in retrograde chronic total occlusion (CTO) percutaneous coronary intervention $(\mathrm{PCl})$. However, there is little evidence regarding the selection of an interventional septal collateral (SC). we aimed to identify the predictors of successful guidewire crossing using clinical and anatomical characteristics. Overall, 216 derivation cases and 86 validation cases that included retrograde CTO $\mathrm{PCl}$ were analyzed. The technical success rate was $79.1 \%$ and there were no significant differences in the Gensini score, SYNTAX score, J-CTO score and Progress Score between two groups. Multivariate logistic regression analysis revealed that diabetes, small size, corkscrew, and side branch at tortuosity were independent factors of success in crossing SCs. We developed a nomogram to predict the success rate, which demonstrates favorable calibration and formed the Sep-CTO score. The calibration and decision curve analysis also demonstrated the reliability and accuracy of this clinical prediction model. The receiver-operating characteristic area of the nomogram was 0.870 . Compared to the aforementioned scoring systems, Sep-СTO score was the most powerful. The nomogram may be a useful clinical tool. We found four independent variables to predict the successful guidewire crossing in septal collaterals.

\section{Introduction}

Chronic total occlusion (CTO) is defined as grade 0 thrombolysis in myocardial infarction (TIMI) flow for more than 3 months, which is frequently encountered during coronary angiography in patients with coronary artery disease (CAD) [1]. Revascularization of CTO can relieve angina, improve heart function, and decrease mortality [2]. According to the hybrid algorithm, retrograde approach is a crucial and effective technique to recanalize CTO when the antegrade attempt has failed or the occlusion has an ambiguous proximal cap, poor distal target, and appropriate collaterals. Septal collaterals (SC) are the most commonly used vessels in the retrograde technique; they form the most important collateral pathway for CTO percutaneous coronary intervention (PCI) [3]. Compared with the complications rate with epicardial collaterals, it is lower with SC [4].

Successful SC guidewire (GW) crossing is the first step in the retrograde technique. The key is to select the collateral vessel with adequate anatomical characteristics. Several groups have reported on the predictive factors of retrograde $\mathrm{CTO} \mathrm{PCl}$ [5]. However, those score systems predicted the success rate of GW crossing through both epicardial and septal collaterals, which lacks the representative for septal approach. Furthermore, the predictors were assigned 1-2 points using logistic regression, which carries low sensitivity and specificity for each patient [6-9].

Currently, nomogram is considered a useful tool to evaluate the risk by integrating important clinical and anatomical characteristics. By creating a direct evaluation system, a predictive nomogram may assist operators in selecting the optimal SC vessel. Therefore, the aim of this study was to establish a nomogram for identifying the clinical and potential angiographic predictors of successful GW crossing the SC vessels in retrograde CTO PCl. 


\section{Results}

\section{Baseline characteristics of participants}

Table 1 summarizes the clinical characteristics of the derivation cohort $(n=216)$ and validation cohort $(n=86)$. The mean age was $57.8 \pm 10.2$ and $58.6 \pm 10.0$ years in these groups, respectively. The incidences of hyperlipidemia, diabetes, stroke, history of $\mathrm{PCl}$, and smoking were not significantly differently between the groups (all $p>0.05$ ). Similarly, there were no statistical significance in the body mass index (BMI), left ventricular ejection fraction, Gensini score, SYNTAX score, J-CTO score, and Progress Score (all $p>0.05$ ). The main angiographic characteristics of the two groups are summarized in Table 1.

In the derivation set, the incidence of diabetes was significantly higher in the failed group than that in the successful group $(p<0.05)$. In terms of angiographic characteristics, the failed group demonstrated significantly higher proportion of small size, corkscrew, and side branch at tortuosity than those in the successful group (all $p<0.05$, Table 2 ).

\section{Univariate and multivariate analysis}

Univariate and multivariate logistic regression analyses were used to explore the association of risk factors with success rate of GW crossing. The univariate analysis demonstrated that diabetes (odds ratio, OR: 2.305), small size (OR: 2.778), corkscrew (OR: 4.028), and side branch at tortuosity (OR: 2.190) were associated with success in GW crossing (all $P<0.05$ ). According to multivariate logistic regression analyses, diabetes, small size, corkscrew, and side branch at tortuosity were identified as independent factors in the training cohort (Table 3, Figure 4).

\section{Novel nomogram score system}

Corkscrew was the biggest influencing factor on the prognosis, whereas diabetes had the least effect. Validation of the nomogram was performed using bootstrap analyses with 1,000 resamples; the internal validation cohorts revealed favorable discrimination of the nomogram, demonstrating that the nomogram could be clinically implemented (Figure 5).

\section{Validation of the predictive accuracy of the nomogram}

Overall, 86 patients were included in the validation cohort between February 2019 and October 2020. The calibration was drawn to evaluate the calibration of the model in the validation set (Figure 6). ROC analyses were used to evaluate the discrimination of the model; the area under the curve (AUC) was 0.870 (95\% confidence interval, Cl: 0.792-0.948, Table 5) and indicated better predictive accuracy. Additionally, the decision curve analysis (DCA) curve demonstrated that the novel nomogram also included a higher clinical net (Figure 7).

\section{Procedure data and clinical outcome}


Table 4 summarizes the procedures and clinical outcome data. Femoral and radial access were used in the majority of patients (91.0\%). The high prevalence of GWs used was Sion (Asahi Intecc, Nagoya, Japan), which was $93.3 \%$. The common final crossing technique included the Reverse CART (49.1\%), RWE (19.7\%), AWE (8.3\%), and parallel wires (5.3\%). Other techniques included kissing wires (10.1\%) and ADR (3.1\%). Furthermore, the technical success rate was $79.1 \%$ and the procedural success rate was $75.5 \%$. For procedural complications and adverse events, the incidence of CC perforation was $4.6 \%$. One patient developed periprocedural myocardial infarction and six patients developed no-flow or slow-flow.

\section{Discriminatory power of different scores}

We also compared the predictive ability of the new model and conventional staging systems by comparing AUC of ROC curves (Table 5; Figure 8). The AUC of Retro-CTO score was 0.698 (95\% Cl: 0.563-0.834, P<0.05) and Figure 8 illustrates Retro-CTO score's probability of success in different groups. The J-Channel score demonstrated intermediate predictive value (AUC: 0.776 ; 95\% Cl: 0.644-0.909; $\mathrm{P}<0.01)$. The predictive value of EPI-score included AUC of $0.702(95 \% \mathrm{Cl}: 0.558-0.846 ; \mathrm{P}<0.05)$. The HLK score demonstrated little predictive value on the septal channel retrograde revascularization (AUC: 0.611; $95 \% \mathrm{Cl}: 0.4614-0.761, \mathrm{P}=0.19)$. Compared to those systems, the Sep-CTO score had the strongest predictive power with success rates of $100 \%, 75 \%$, and $50 \%$ for easy, intermediate, and difficult groups, respectively (Figure 9). Additionally, the Sep-CTO score may predict the GW crossing time as well (Figure 10).

\section{Discussion}

In this study, the demographic, clinical, and SC anatomical characteristics were analyzed to examine the association between the predictive factors and success with SC GW crossing. New predictive models including a nomogram based on multiple logistic regression were developed and validated. To the best of our knowledge, this is the first attempt to develop a diagnostic method for SC GW crossing based on a nomogram. Our results provide a new perspective on the relevant predictive factors with better discriminatory power. Diabetes, small size, corkscrew, and side branch at tortuosity were significant predictors of success in SC GW crossing (Figure 11).

Despite remarkable progress in devices, techniques, and experiences over the decades, the recanalization rate of CTO is still low and represents the "final frontier" of PCI [19]. The retrograde technique can improve the success rate of CTO PCI when the antegrade GW cannot reach the true lumen of the occlusion distally. The first retrograde crossing via SC was performed by Surmely in 2009 [20]. Regarding the anatomical distribution of collaterals, the left anterior descending septal collaterals to the posterior descending artery is the common in right coronary artery СTO, and we also found a high frequency of septal collaterals from the posterior descending artery to the left anterior descending in left anterior descending CTO [18]. However, SC are preferred due to higher success rate and lower risk of perforation and tamponade compared to epicardial and saphenous vein grafts [21]. Finding an adequate vessel can improve the operative efficiency and decrease the dose of radiation exposure. In our study, we identified 
four independent risk factors-diabetes, small size, corkscrew, and side branch at tortuosity-using univariate and multivariate analyses as predictors of successful GW crossing.

Epidemiological evidence indicate that diabetes is a crucial risk factor in cardiovascular diseases. Patients with CTO with diabetes often have reduced coronary collateralization through multiple mechanisms, including decrease pressure gradient, oxidative stress, and inhibition of proangiogenic factors [22]. In this study, compared to the successful group, the prevalence of diabetes was higher in the failed group, which may be associated with more invisible coronary collaterals and difficult to cross with the surfing technique. The small vessels include Werner $\mathrm{CCO}$ in which surfing $\mathrm{CC}$ crossing techniques are commonly used. However, controversy remains in this field. Dautov et al. found that invisible SC were crossed with as much success as larger CCs and faster [23]. A previous study enrolled 216 patients with retrograde CTO in whom $\mathrm{PCl}$ was attempted and revealed collateral channel size as a predictor of failure [9]. We found that small size was an independent factor related to failure of GW crossing in SC. Corkscrew was the most powerful prediction variable of GW crossing in our study. From our experience, GW crossing is difficult in a corkscrew vessel, especially, if it is small in size. Additionally, manipulation of GW was poor in the collateral channel, especially, the distal segment. When the septal collateral was corkscrew-shaped, it was difficult to prevent perforation. In contrast, the tip of GW is difficult to keep angle in corkscrew. Huang et al. proved that side branch at CCs tortuosity was an independent predictor of procedure success in epicardial collaterals [7]. Similarly, side branch at CCs tortuosity was also significantly associated with GW tracking in SC. This approach resulted in difficult advancement of GW. Besides, due to a lack of support from the catheter, GW steerability is inadequate.

The important predictor of GC crossing included in the nomogram was the Sep-CTO score. Recently, some researchers have focused on the assessment of GW tracking. Scoring systems have been constructed to predict the success of GW tracking in the retrograde approach, such as EPI-score, RetroCTO score, J-channel score, and HLK score. EPI-score demonstrated that CC tortuosity, side branch at CCs tortuosity, inadequate CCs size, and inadequate CCs exit location were independent predictors associated with technical success in the epicardial channel [7]. Other studies have highlighted that those the score had a significant predicting ability in collateral channels. Consistent with the above results, our singlecenter study found that the Sep-CTO score was a reliable system in the prediction of successful GW crossing in the septal channel, which also might indicate the GW crossing time. Therefore, given the anatomical features, the assessment of the collateral channels is critical.

In the ERCTO registry, the success rate of GW crossing the collateral channel was $75.3 \%$ and the clinical success rate was $71.2 \%$ [24]. In our study, retrograde wire advancement to the distal CTO cap through SP was successful in $79.1 \%$ of cases, and the rate of retrograde procedural success was $75.5 \%$. Additionally, perforations were observed 14 cases; they were minor and asymptomatic. These findings confirm that septal CCs are safer and, therefore, should be preferred.

\section{Material And Methods}




\section{Study Patient and Data Collection}

Overall, 216 inpatients with chest pain who underwent attempted retrograde CTO PCl via SC by highvolume operators [10] (>75 total CTO PCls and $>20$ retrograde attempts overall) between January 2016 and January 2019 at The Anzhen Hospital were consecutively enrolled. Between February 2019 and October 2020, an independent cohort of consecutive patients was prospectively enrolled using the same inclusion and exclusion criteria. These patients formed the validation cohort in this study. The patients were assigned to the successful $(n=167)$ or failed $(n=49)$ group based on the outcome of the procedure (Figure 1).

Baseline, procedural, and hospitalization data were recorded. The Gensini score [11], SYNTAX score (Synergy between Percutaneous Coronary Intervention with TAXUS and Cardiac Surgery) [12], J-CTO score (Multicenter CTO Registry in Japan) [13], and Progress Score (Prospective Global Registry for the study of СTO intervention) [14] were calculated by two experienced interventional cardiologists. The study protocol was approved by the Institutional Review Board of The Anzhen Hospital, Beijing, China.

\section{Definitions}

CTO was defined as an occlusion lasting for more than 3 months based on the first onset of angina pectoris, previous angiogram, or previous infarction and TIMI grade 0 . All procedures in this study followed the hybrid algorithm.

The diagnostic criteria for the classical risk factors, including dyslipidemia [15], hypertension [16], and diabetes [17], were based on authoritative international guidelines. Two experienced interventional cardiologists collected the anatomic characteristics of SC (Figure 2). According to McEntegart MB et al [18]. CC entry angle $<90^{\circ}$ was described as an acute of $\angle 90^{\circ}$ between $\mathrm{CC}$ and the recipient vessel. CC exit angle $<90^{\circ}$ was defined as the angle was less than $90^{\circ}$ at channel exit. Reverse bend was defined as part of the bend folded at an angle of more than $90^{\circ}$. Continuous bends included more than three successive curves of more than $180^{\circ}$. Corkscrew was described as three or more continuous bends with a ratio of vessel amplitude/vessel diameter (AD ratio) $\leq 2$. Side branch at tortuosity was defined as bifurcation within a tortuous part of CC. Small size of the vessels was assessed using Werner's CC score and was defined as $\mathrm{CCO}$ or $\mathrm{CC} 1$ (Figure 2,3).

Retro-CTO score was developed by Chai et al.; it includes the Werner's score, diameter of distal CTO lesion ( $<1.5 \mathrm{~mm}$ ), and tortuous collateral [6]. EPI-score was developed by Huang et al.; it includes tortuosity, side branch at tortuosity, inadequate size of CCs, and inadequate exit location of CCs [7]. J-channel score includes the vessel size, reverse bend, continuous bends, and corkscrew; it was developed by Nagamatsu et al. in 2020 [8]. HLK score consists of vessel size and tortuosity and was developed by Hsien-Li et al [9].

Successful GW crossing was defined as the guide wire crossing through the SC from the retrograde side and reaching the distal vessel segment. Technical success was defined as successful CTO recanalization 
with $<20 \%$ residual stenosis and TIMI flow grade 3 along with the absence of in-hospital adverse events.

\section{Statistical Methods}

Continuous variables are reported as mean \pm standard deviation for normally distributed data or median and quartiles (Q1-Q3) for non-normally distributed data. Discrete variables are expressed as frequency and percentage and compared using the chi-squared test. Logistic regression analysis was performed to identify factors. For further analysis, a nomogram was formulated based on the results of multivariable logistic regression analysis. For validating the nomogram, the calibration curves were calculated from the multivariate logistic model. Bootstrapping with 1000 resamples was used for these analyses. Decision curve analysis (DCA) was performed to determine the clinical usefulness of the nomogram for derivation and validation sets. We also performed receiver-operating characteristic (ROC) curve analyses to compare the diagnostic performance of each scoring system in the validation sets. A two-sided $p$-value $<0.05$ was considered statistically significant. The statistical computations were performed using SPSS v23.0 (IBM Inc., Armonk, NY, USA), STATA version 16.0 (StataCorp, College Station, TX, USA), and R v3.6.2 (R Foundation for statistical Computing, Vienna, Austria).

\section{Ethics approval and consent to participate.}

The protocol of this study and informed consent of all participants were approved by the Ethics Institutional Review Board of The Anzhen Hospital, Beijing, China. All methods were carried out in accordance with relevant guidelines and regulations, besides,informed consent was obtained from all subjects.

\section{Consent for publication.}

Authors give full consents for publication of this present article.

\section{Limitations}

Our study had some limitations. First, some selection bias was inevitable due to the retrospective design and single-center nature of the study. Second, the operator's experience plays a crucial role in CTO PCl; this study included high-volume operators. Therefore, this scoring system may not be applicable to beginners. Finally, our nomogram may be a useful non-laboratory clinical tool. Further prospective multicenter studies are required to assess its acceptability and usability.

\section{Conclusion}

To our knowledge, this is the first application of logistic regression to produce a nomogram in predicting the success of GW crossing in retrograde CTO PCI in China. The Sep-CTO score can be used for judging the difficulty of GW crossing and carries strong translatability to clinical applications.

\section{Declarations}




\section{Authors' contributions}

Tong Liu for wrote article; Yuchao Zhang for collect data; Zheng Wu for guide; Yun Lv for analysis; Jinghua Liu for review the article.

\section{Additional Information}

\section{Funding}

The study was funded by the National Natural Science Foundation of China (81970291).

\section{Competing interests}

None

\section{Data availability}

availability

\section{Ethics approval}

Yes

\section{References}

1. Tsai, T. et al. Long term clinical impact of successful recanalization of chronic total occlusion in patients with and without type 2 diabetes mellitus. Cardiovasc Diabetol. 19, 119 (2020).

2. Ybarra, F. \&Rinfret, S. Why and how should we treat chronic total occlusion? Evolution of state-of-theart methods and future directions. Can. J. Cardiol. 10.1016/j.cjca.2020.10.005 (2020).

3. Brilakis, S. et al. A percutaneous treatment algorithm for crossing coronary chronic total occlusions. JACC. Cardiovasc Interv. 5,367-379 (2012) .

4. Lee,K. et al. Retrograde approach is as effective and safe as antegrade approach in contemporary percutaneous coronary intervention for chronic total occlusion: a Taiwan single-center registry study. Acta Cardiol Sin. 33,20-27 (2017).

5. Yamaji K. et al. Retrograde-angioscopy guided wiring technique in chronic total occlusion lead to successful revascularization. J Cardiol Cases. 22,110-113 (2020).

6. Chai,L. et al. Clinical prediction score for successful retrograde procedure in chronic total occlusion percutaneous coronary intervention. Cardiology. 134,331-339 (2016).

7. Huang,et al. Epicardial collateral channel for retrograded recanalization of chronic total occlusion percutaneous coronary intervention: predictors of failure and procedural outcome. $J$ Interv Cardiol. 31, 23-30 (2018). 
8. Nagamatsu,et al. Successful guidewire crossing via collateral channel at retrograde percutaneous coronary intervention for chronic total occlusion: the J-Channel score. Eurolntervention. 15, e1624e1632 (2020).

9. Huang,C. et al. Collateral channel size and tortuosity predict retrograde percutaneous coronary intervention success for chronic total occlusion. Circ Cardiovasc Interv. 11,e005124 (2018).

10. Kleiman,S. et al. Should interventional cardiologists super-subspecialize? Moving from patient selection to operator selection. JACC Cardiovasc Interv. 14, 97-100 (2021).

11. Rampidis,P. et al. A guide for Gensini Score calculation. Atherosclerosis. 287, 181-183 (2019).

12. Serruys,W. et al. Did the SYNTAX score pass the test of time? JACC Cardiovasc Interv. 13, 1207-1210 (2020).

13. Morino, et al. Predicting successful guidewire crossing through chronic total occlusion of native coronary lesions within 30 minutes: the J-CTO (Multicenter CTO Registry in Japan) score as a difficulty grading and time assessment tool. JACC Cardiovasc Interv. 4, 213-221 (2011).

14. Danek,A. et al. Development and validation of a scoring system for predicting periprocedural complications during percutaneous coronary interventions of chronic total occlusions: the Prospective Global Registry for the Study of Chronic Total Occlusion Intervention (PROGRESS CTO) complications score. J Am Heart Assoc. 5, e004272 (2016).

15. Jellinger,S. et al. American Association of Clinical Endocrinologists and American College of Endocrinology guidelines for management of dyslipidemia and prevention of cardiovascular disease. Endocr Pract. 23, 1-87 (2017).

16. Williams, et al. $2018 \mathrm{ESC} / \mathrm{ESH}$ Guidelines for the management of arterial hypertension. $G$ Ital Cardiol. 39, 3021-3104 (2018).

17. Garber,J. et al. Consensus statement by the American association of clinical endocrinologists and American college of endocrinology on the comprehensive type 2 diabetes management algorithm 2020 executive summary. Endocr Pract. 26, 107-139 (2020).

18. McEntegart,B. et al. The collateral circulation of coronary chronic total occlusions. Eurolntervention. 11, e1596-e1603 (2016).

19. Yeh,W. et al. Depression and angina among patients undergoing chronic total occlusion percutaneous coronary intervention: the OPEN-CTO registry. JACC Cardiovasc Interv. 12,651-658 (2019).

20. Tajti, et al. Technical and procedural outcomes of the retrograde approach to chronic total occlusion interventions. Eurolntervention. 1611, e891-e899 (2020).

21. Benincasa, et al. Outcomes of the retrograde approach through epicardial versus non-epicardial collaterals in chronic total occlusion percutaneous coronary intervention. Cardiovasc Revasc Med. 18, 393-398 (2017).

22. Shen, et al. Reduced coronary collateralization in type 2 diabetic patients with chronic total occlusion. Cardiovasc Diabetol. 17, 26 (2018). 
23. Dautov, et al. Safety and effectiveness of the surfing technique to cross septal collateral channels during retrograde chronic total occlusion percutaneous coronary intervention. Eurolntervention. 12,e1859-e1867 (2017).

24. Galassi,R. et al.Retrograde recanalization of chronic total occlusions in Europe: procedural, inhospital, and long-term outcomes from the multicenter ERCTO registry. J Am Coll Cardiol. 65, 23882400 (2015).

\section{Tables}

Table 1. Clinical and angiographic characteristics in the derivation and validation sets. 


\begin{tabular}{|c|c|c|c|c|}
\hline \multirow[t]{2}{*}{ Variable } & Derivation set & Validation set & \multirow[t]{2}{*}{$t / Z$} & \multirow[t]{2}{*}{ P-value } \\
\hline & $(n=216)$ & $(n=86)$ & & \\
\hline Age (years) & $57.8 \pm 10.2$ & $58.6 \pm 10.0$ & 0.615 & 0.539 \\
\hline $\mathrm{BMI}\left(\mathrm{kg} / \mathrm{m}^{2}\right)$ & $26.2 \pm 3.8$ & $26.4 \pm 4.6$ & 0.271 & 0.787 \\
\hline Male (\%) & $164(75.9)$ & $66(76.7)$ & 0.023 & 0.880 \\
\hline Diabetes (\%) & $62(28.7)$ & $22(25.6 \%)$ & 0.299 & 0.585 \\
\hline Hypertension (\%) & $138(63.9)$ & $58(67.4)$ & 0.341 & 0.559 \\
\hline Dyslipidemia (\%) & $137(63.4)$ & $52(60.5)$ & 0.23 & 0.631 \\
\hline Smoking (\%) & $84(38.9)$ & $31(36.0)$ & 0.211 & 0.646 \\
\hline Stroke (\%) & $24(11.1)$ & $10(11.6)$ & 0.016 & 0.898 \\
\hline LVEF (\%) & $60.7 \pm 8.3$ & $60.7 \pm 9.4$ & 0.027 & 0.978 \\
\hline History of $\mathrm{PCl}(\%)$ & 77 (35.6) & $33(38.4)$ & 0.197 & 0.657 \\
\hline \multicolumn{5}{|l|}{ Target CTO vessel } \\
\hline RCA (\%) & $133(61.6)$ & $50(58.1)$ & 0.304 & 0.581 \\
\hline LAD (\%) & $83(38.4)$ & $36(41.9)$ & 0.304 & 0.581 \\
\hline \multicolumn{5}{|l|}{ Score systems } \\
\hline Gensini score & $54(45,64)$ & $52(43,64)$ & -0.624 & 0.531 \\
\hline SYNTAX score & $22(19,25)$ & $21(19,24)$ & -1.319 & 0.187 \\
\hline J-CTO score & $2.0 \pm 1.0$ & $2.1 \pm 1.0$ & 1.059 & 0.660 \\
\hline Progress Score & $1.0(0,1.0)$ & $1.0(0,1.0)$ & -1.014 & 0.311 \\
\hline \multicolumn{5}{|l|}{ Angiographic characteristics } \\
\hline SC entry angle $<90^{\circ}(\%)$ & $14(6.5)$ & $6(7.0)$ & 0.024 & 0.876 \\
\hline SC exit angle $<90^{\circ}(\%)$ & $17(7.9)$ & $7(8.1)$ & 0.006 & 0.938 \\
\hline Small size (\%) & $50(23.1)$ & $12(14.0)$ & 3.187 & 0.074 \\
\hline Inadequate SC exit location (\%) & $34(15.7)$ & $12(14.0)$ & 0.152 & 0.696 \\
\hline Reverse bend (\%) & $23(10.6)$ & $5(5.8)$ & 1.709 & 0.191 \\
\hline Continuous bends (\%) & $35(16.2)$ & $9(10.5)$ & 1.627 & 0.202 \\
\hline Corkscrew (\%) & $66(30.6)$ & $23(26.7)$ & 0.43 & 0.512 \\
\hline Side branch at tortuosity (\%) & $60(27.8)$ & $21(24.4)$ & 0.354 & 0.552 \\
\hline
\end{tabular}


BMI: body mass index; LVEF: left ventricular ejection fraction; PCI: percutaneous coronary intervention; RCA: right coronary artery; LAD: left anterior descending; SC: septal collateral.

Table 2. Clinical and angiographic characteristics in the derivation sets. 


\begin{tabular}{|c|c|c|c|c|}
\hline \multirow[t]{2}{*}{ Variable } & successful group & failed group & \multirow[t]{2}{*}{$t / Z$} & \multirow[t]{2}{*}{ P-value } \\
\hline & $(n=167)$ & $(n=49)$ & & \\
\hline Age (years) & $57.7 \pm 10.2$ & $58.4 \pm 10.2$ & -0.377 & 0.706 \\
\hline BMI $\left(\mathrm{kg} / \mathrm{m}^{2}\right)$ & $26.3 \pm 3.8$ & $25.9 \pm 3.9$ & 0.569 & 0.570 \\
\hline Male (\%) & $129(77.2)$ & 35 (71.4) & 0.701 & 0.402 \\
\hline Diabetes (\%) & $41(24.6)$ & $21(42.9 \%)$ & 6.204 & 0.013 \\
\hline Hypertension (\%) & $104(62.3)$ & $34(69.4)$ & 0.831 & 0.362 \\
\hline Dyslipidemia (\%) & $103(61.7)$ & $34(69.4)$ & 0.971 & 0.324 \\
\hline Smoking (\%) & $62(37.1)$ & $22(44.9)$ & 0.211 & 0.646 \\
\hline Stroke (\%) & 19 (11.4) & $5(10.2)$ & 0.053 & 0.818 \\
\hline LVEF (\%) & $60.7 \pm 8.0$ & $60.5 \pm 9.4$ & 0.154 & 0.877 \\
\hline History of $\mathrm{PCl}(\%)$ & $58(34.7)$ & $19(38.8)$ & 0.27 & 0.603 \\
\hline \multicolumn{5}{|l|}{ Target CTO vessel } \\
\hline RCA (\%) & 105 (62.9) & $28(57.1)$ & 0.526 & 0.468 \\
\hline LAD (\%) & $62(37.1)$ & $21(42.9)$ & 0.526 & 0.468 \\
\hline \multicolumn{5}{|l|}{ Score systems } \\
\hline Gensini score & $54(46,64)$ & $56(46,68)$ & -0.336 & 0.737 \\
\hline SYNTAX score & $22(19,25)$ & $23(12,31)$ & -0.349 & 0.727 \\
\hline J-CTO score & $2.0 \pm 1.0$ & $2.0 \pm 1.0$ & 0.743 & 0.459 \\
\hline Progress Score & $1.0(0,1.0)$ & $1.0(0,1.0)$ & -1.434 & 0.152 \\
\hline \multicolumn{5}{|l|}{ Angiographic characteristics } \\
\hline SC entry angle $\mathbb{9} 90^{\circ}(\%)$ & $10(6.0)$ & $4(8.2)$ & 0.296 & 0.587 \\
\hline SC exit angle $\otimes 90^{\circ}(\%)$ & $11(6.6)$ & $6(12.2)$ & 1.673 & 0.196 \\
\hline Small size (\%) & $31(18.6)$ & $19(38.8)$ & 8.7 & 0.003 \\
\hline Inadequate SC exit location (\%) & $24(14.4)$ & $10(20.4)$ & 1.041 & 0.308 \\
\hline Reverse bend (\%) & $16(9.6)$ & 7 (14.3) & 0.881 & 0.348 \\
\hline Continuous bends (\%) & $27(16.2)$ & $8(16.3)$ & 0.001 & 0.979 \\
\hline Corkscrew (\%) & $39(23.4)$ & $27(55.1)$ & 17.996 & 0.001 \\
\hline Side branch at tortuosity (\%) & $40(24.0)$ & $20(40.8)$ & 5.371 & 0.020 \\
\hline
\end{tabular}


BMI: body mass index; LVEF: left ventricular ejection fraction; PCl: percutaneous coronary intervention; RCA: right coronary artery; LAD: left anterior descending; SC: septal collateral.

Table 3. Univariate and multivariate logistic regression analyses for predictors of successful guidewire crossing.

\begin{tabular}{|c|c|c|c|c|}
\hline \multirow[t]{2}{*}{ Variables } & \multicolumn{2}{|l|}{ Univariable } & \multicolumn{2}{|l|}{ Multivariable } \\
\hline & OR $(95 \% \mathrm{Cl})$ & P-value & OR $(95 \% \mathrm{Cl})$ & $P$-value \\
\hline $\mathrm{BMI}, \mathrm{kg} / \mathrm{m}^{2}$ & $0.974(0.891-1.066)$ & 0.568 & - & - \\
\hline Diabetes & $2.305(1.183-4.489)$ & 0.014 & $2.604(1.238-5.481)$ & 0.012 \\
\hline Hypertension & $1.408(0.711-2.788)$ & 0.326 & - & - \\
\hline Smoking & $1.380(0.724-2.629)$ & 0.327 & - & - \\
\hline History of PCl & $1.190(0.617-2.296)$ & 0.603 & - & - \\
\hline SC entry angle $₫ 90^{\circ}$ & $1.396(0.418-4.661)$ & 0.588 & - & - \\
\hline SC exit angle $₫ 90^{\circ}$ & $1.979(0.692-5.657)$ & 0.203 & - & - \\
\hline Small size & $2.778(1.387-5.565)$ & 0.004 & $3.990(1.763-9.028)$ & 0.001 \\
\hline Inadequate SC exit location & $1.528(0.674-3.463)$ & 0.310 & - & - \\
\hline Reverse bend & $1.573(0.607-4.074)$ & 0.351 & - & - \\
\hline Continue bend & $1.012(0.427-2.396)$ & 0.979 & - & - \\
\hline Corkscrew & $4.028(2.067-7.851)$ & $₫ 0.001$ & $4.087(1.993-8.382)$ & $\otimes 0.001$ \\
\hline Side branch at tortuosity & $2.190(1.119-4.285)$ & 0.022 & $3.036(1.375-6.703)$ & 0.006 \\
\hline J-CTO score & $0.871(0.605-1.254)$ & 0.457 & - & - \\
\hline Progress Score & $0.714(0.415-1.228)$ & 0.223 & - & - \\
\hline SYNTAX score & $0.995(0.909-1.089)$ & 0.995 & - & - \\
\hline Gensini score & $1.003(0.983-1.023)$ & 0.771 & - & - \\
\hline
\end{tabular}

BMI: body mass index; PCI: percutaneous coronary intervention; SC: septal collateral.

Table 4. Procedure and complication outcomes. 


\begin{tabular}{|ll|}
\hline Procedure Date ( $\mathrm{n}=302)$ & \\
\hline Vascular access & \\
\hline Femoral and femoral (\%) & $25(8.2)$ \\
\hline Fermoral and radial (\%) & $275(91.0)$ \\
\hline Radial and radial (\%) & $2(0.8)$ \\
\hline Successful guidewire crossing CC (n=239) & \\
\hline Sion & $191(79.9)$ \\
\hline Fielder series & $32(13.4)$ \\
\hline Sionblue & $6(2.5)$ \\
\hline Suoh03 & $10(4.2)$ \\
\hline Final Crossing technique ( $\mathrm{n}=228)$ & \\
\hline Reverse CART (\%) & $112(49.1)$ \\
\hline RWE (\%) & $45(19.7)$ \\
\hline Kissing wires (\%) & $23(10.1)$ \\
\hline AWE (\%) & $19(8.3)$ \\
\hline Parallel wires (\%) & $12(5.3)$ \\
\hline ADR (\%) & $7(3.1)$ \\
\hline Others (\%) & $10(5.3)$ \\
\hline GW Crossing success (\%) & $239.7 \pm(79.1)$ \\
\hline Technical success (\%) & $228(75.5)$ \\
\hline Contrast volume (mL) & $331.6 \pm 130.3$ \\
\hline Radiation dose (mGy) & \\
\hline Fluoroscopy time (min) & \\
\hline guidewire cross SC time (min) & \\
\hline Total procedure time (min) & \\
\hline Procedural complication and adverse event & \\
\hline SCronary perforation (\%) & \\
\hline
\end{tabular}




\begin{tabular}{|ll|} 
Periprocedural myocardial infarction (\%) & $1(0.3)$ \\
\hline No-flow/slow-flow (\%) & $6(2.0)$ \\
\hline Ventricular fibrillation (\%) & $1(0.3)$ \\
\hline Death (\%) & $0(0)$ \\
\hline
\end{tabular}

Table 5. ROC Curve analysis of Sep-CTO score, Retro-CTO score, J-Channel score, EPI-score, and HLK score.

\begin{tabular}{|llllllllllll|}
\hline Factors & AUC & P & $95 \% \mathrm{Cl}$ & $\begin{array}{l}\mathrm{Se} \\
(\%)\end{array}$ & $\begin{array}{l}\mathrm{Sp} \\
(\%)\end{array}$ & LR+ & LR- & OR & YI & $\begin{array}{l}\text { Cut- } \\
\text { off } \\
\text { point }\end{array}$ \\
\hline $\begin{array}{l}\text { Sep-CTO } \\
\text { Score }\end{array}$ & 0.870 & $\begin{array}{l}q \\
0.001\end{array}$ & $\begin{array}{l}0.792- \\
0.948\end{array}$ & 0.929 & 0.708 & 3.18 & 0.10 & 31.8 & 0.637 & 89 \\
\hline $\begin{array}{l}\text { Retro-CTO } \\
\text { score }\end{array}$ & 0.698 & 0.019 & $\begin{array}{l}0.563- \\
0.834\end{array}$ & 0.429 & 0.792 & 2.06 & 0.72 & 2.86 & 0.278 & 0.5 \\
\hline $\begin{array}{l}\text { J-Channel } \\
\text { score }\end{array}$ & 0.776 & 0.001 & $\begin{array}{l}0.644- \\
0.909\end{array}$ & 0.857 & 0.611 & 2.20 & 0.23 & 9.57 & 0.468 & 0.5 \\
\hline EPI-score & 0.702 & 0.017 & $\begin{array}{l}0.558- \\
0.846\end{array}$ & 0.643 & 0.736 & 2.44 & 0.49 & 4.98 & 0.379 & 1.5 \\
\hline HLK score & 0.611 & 0.190 & $\begin{array}{l}0.461- \\
0.761\end{array}$ & 0.347 & 0.929 & 4.89 & 0.70 & 6.99 & 0.276 & 2.5 \\
\hline
\end{tabular}

\section{Figures}


41236 patients underwent percutaneous coronary intervention at anzhen hospital between 2016 and 2020

\section{6 consecutive patients with at lease one CTO lesion}

\section{Inclusion criteria:}

- followed the hybrid algorithm

- double angiography

- retrograde attempt in septal collateral

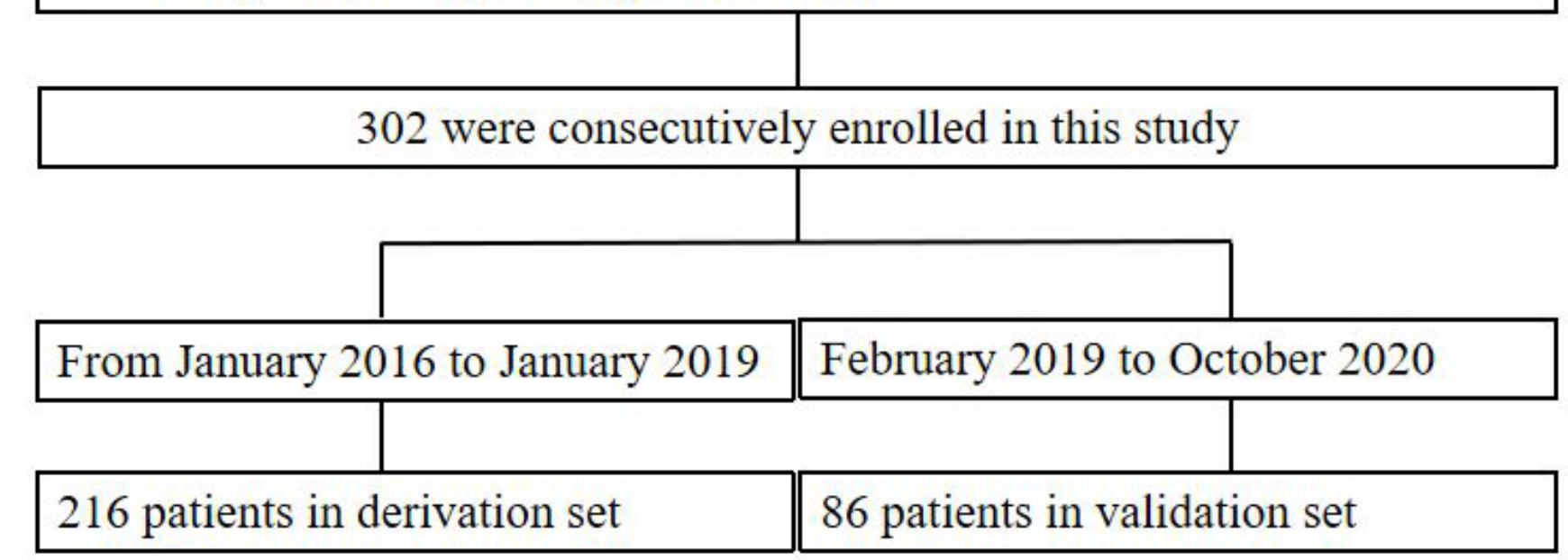

\section{Figure 1}

Population flowchart of retrograde CTO PCI. CTO, chronic total occlusion; PCl, percutaneous coronary intervention. 


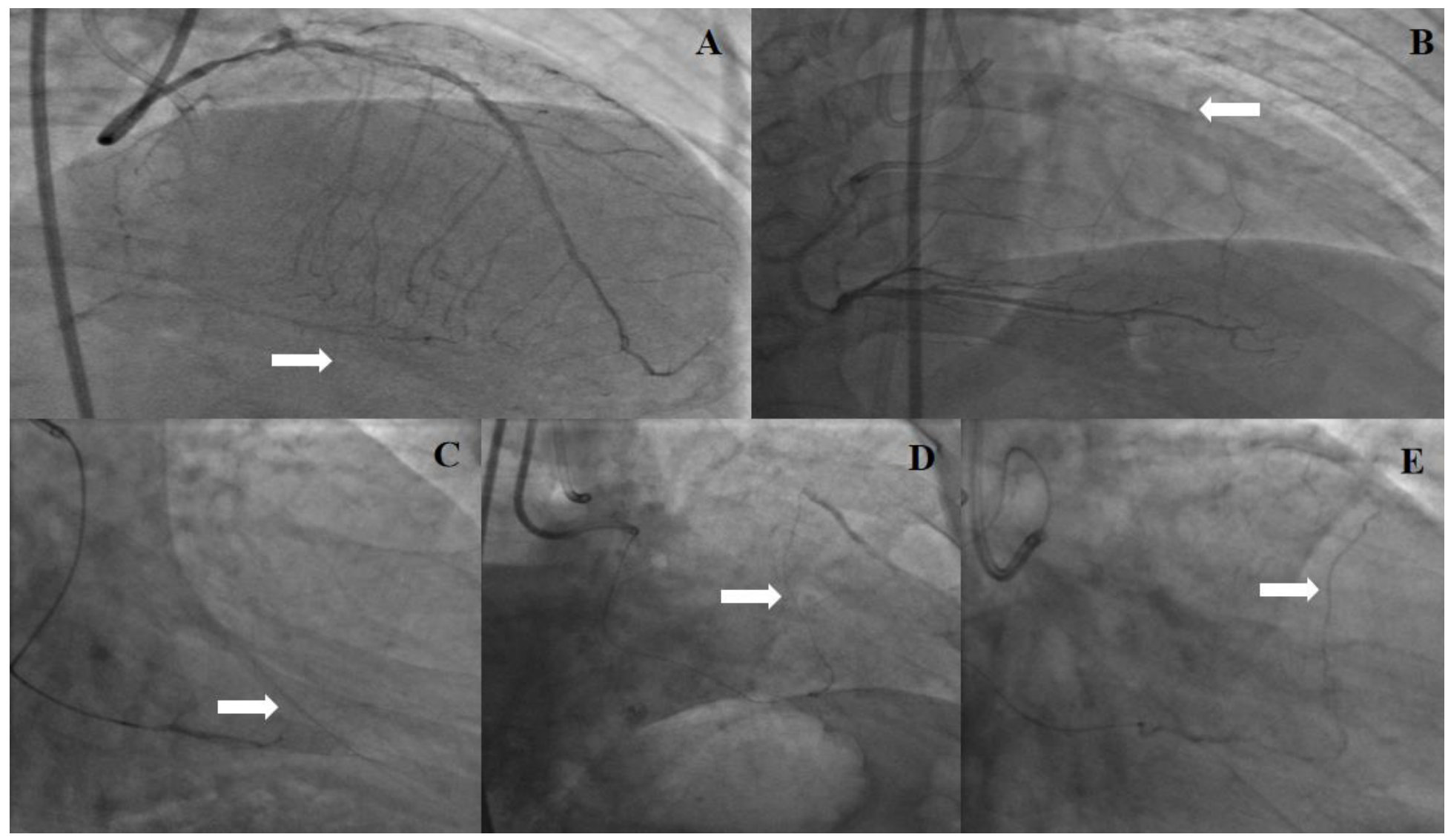

Figure 2

Septal collaterals and Werner's CC score. A, From the left anterior descending to the right coronary artery. $B$, From the right coronary artery to the left anterior descending. C, Werner's CC score 0 . D, Werner's CC score 1. E, Werner's CC score 2. 


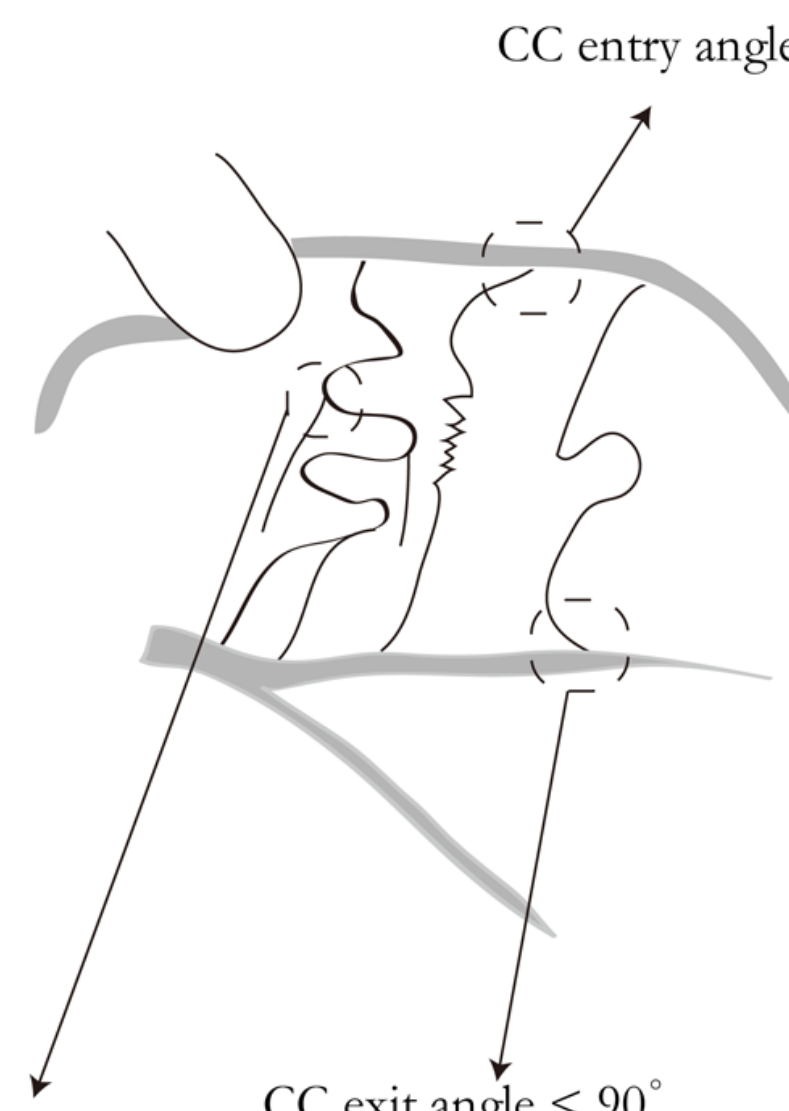

CC exit angle $<90^{\circ}$

Side branch at tortuosity

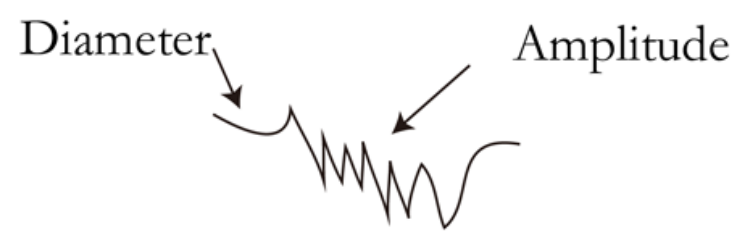

Corkscrew: amplitude/diameter $\leqslant 2$

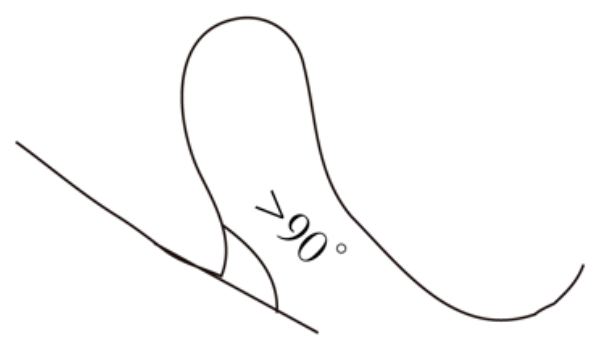

Reverse bend: an angle $>90^{\circ}$

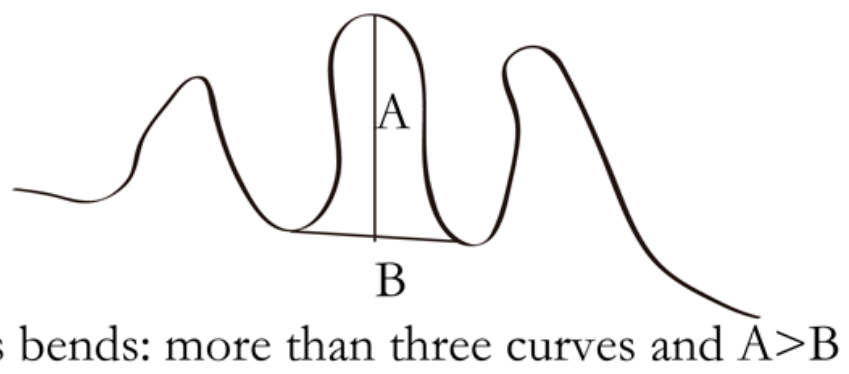

Figure 3

Anatomic characteristics of the septal collateral vessels.

Adjusted $O R(95 \%$ CI $) \quad P$ Value

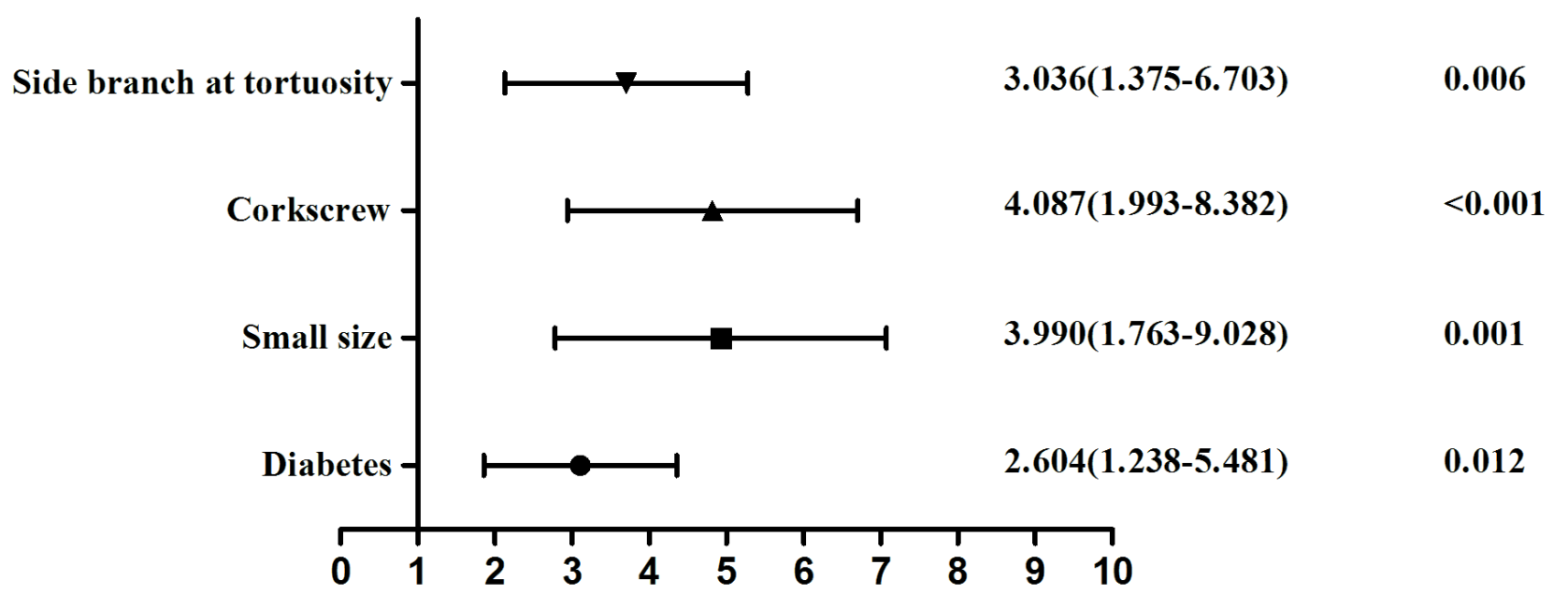

Figure 4

Forest plot of guidewire crossing success. 


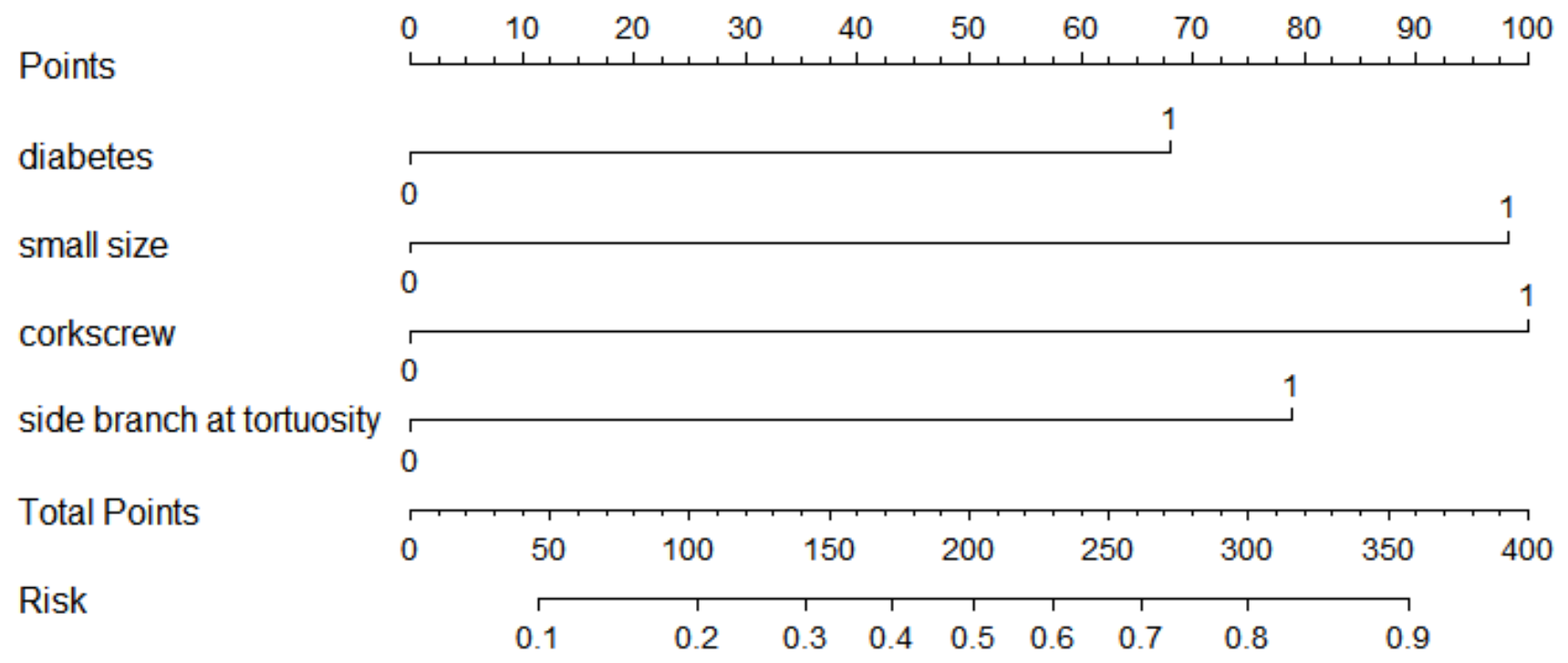

\section{Figure 5}

A nomogram prediction model for the probability of guidewire crossing.

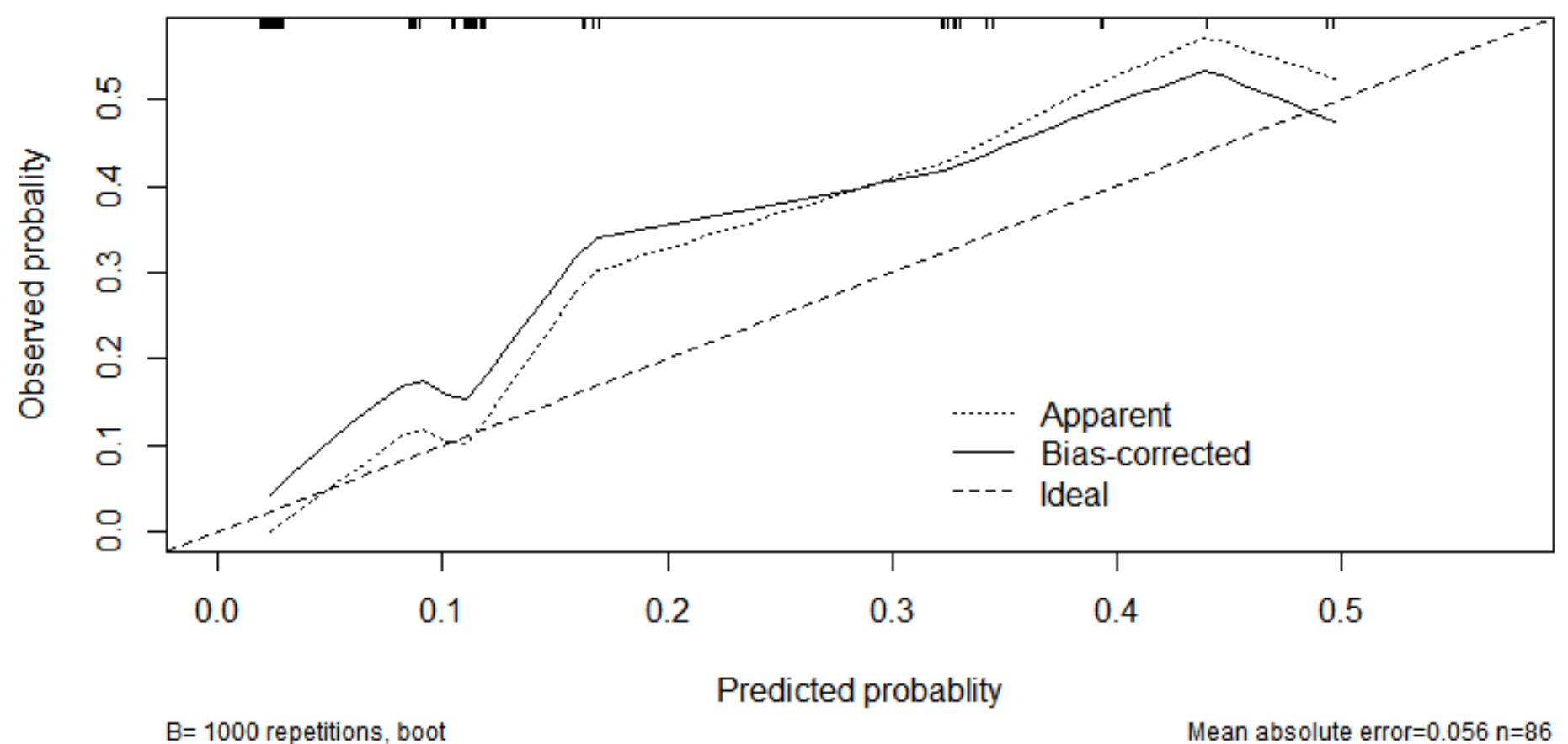

Figure 6 
Calibration curves of the nomogram for predicting guidewire crossing in the validation cohort.
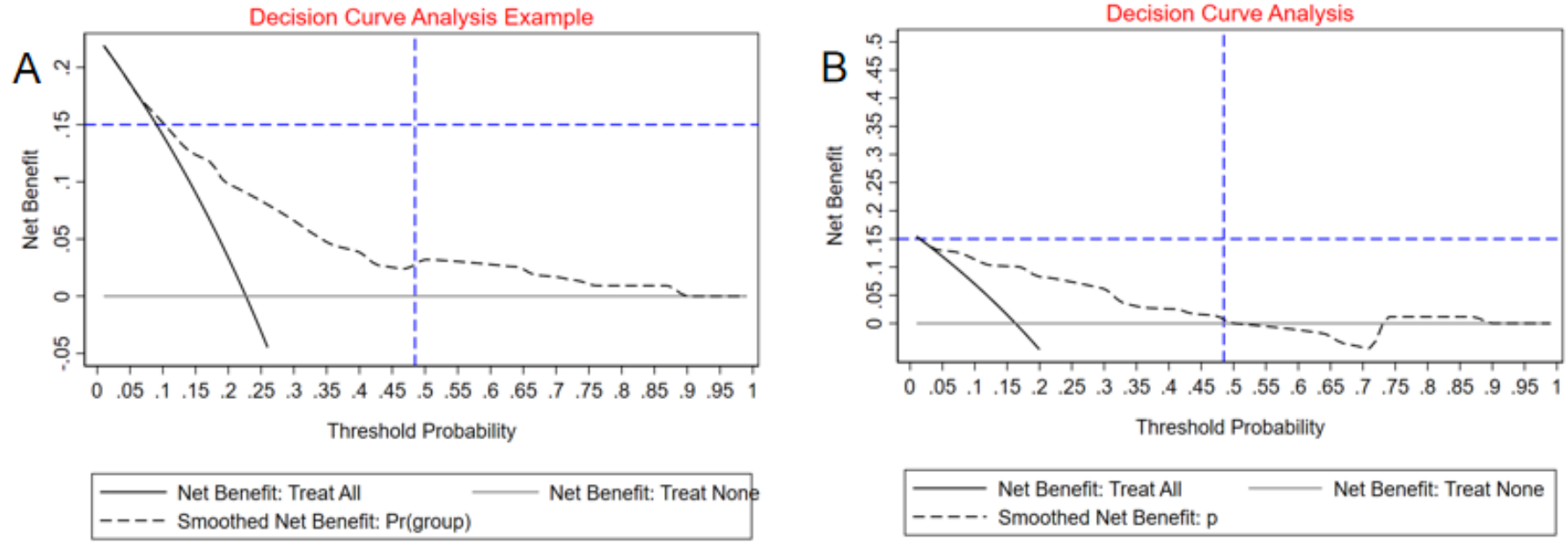

\section{Figure 7}

Decision curve analysis (DCA) of the nomogram for predicting successful guidewire crossing in the derivation cohort $(A)$ and validation cohort $(B)$.

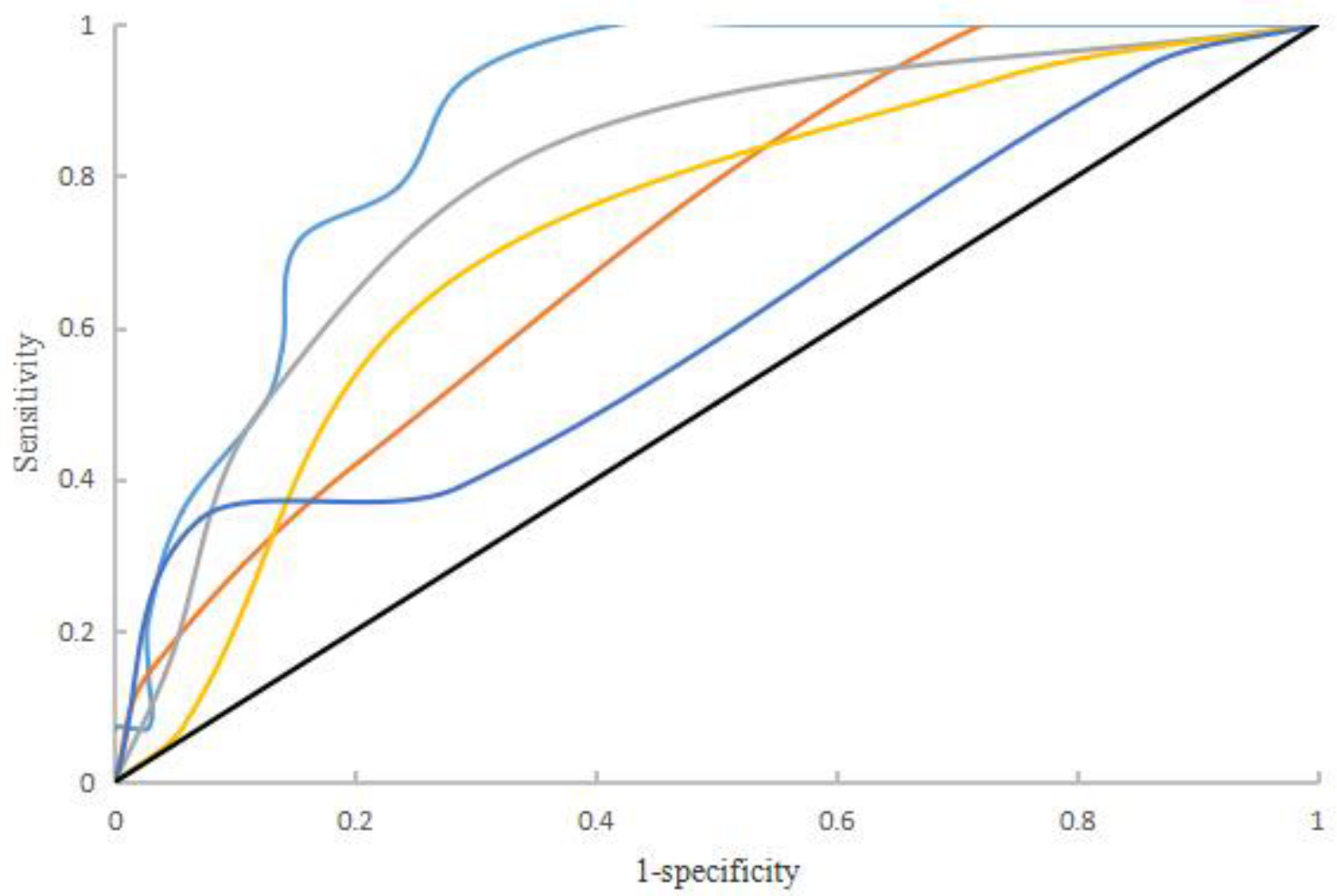

-Sep-CTO score - Retro-CTO score - J-Channel score - EPI-score - HLK score 
ROC curve for the five CTO scores. ROC, receiver-operating characteristics; CTO, chronic thrombotic occlusion.
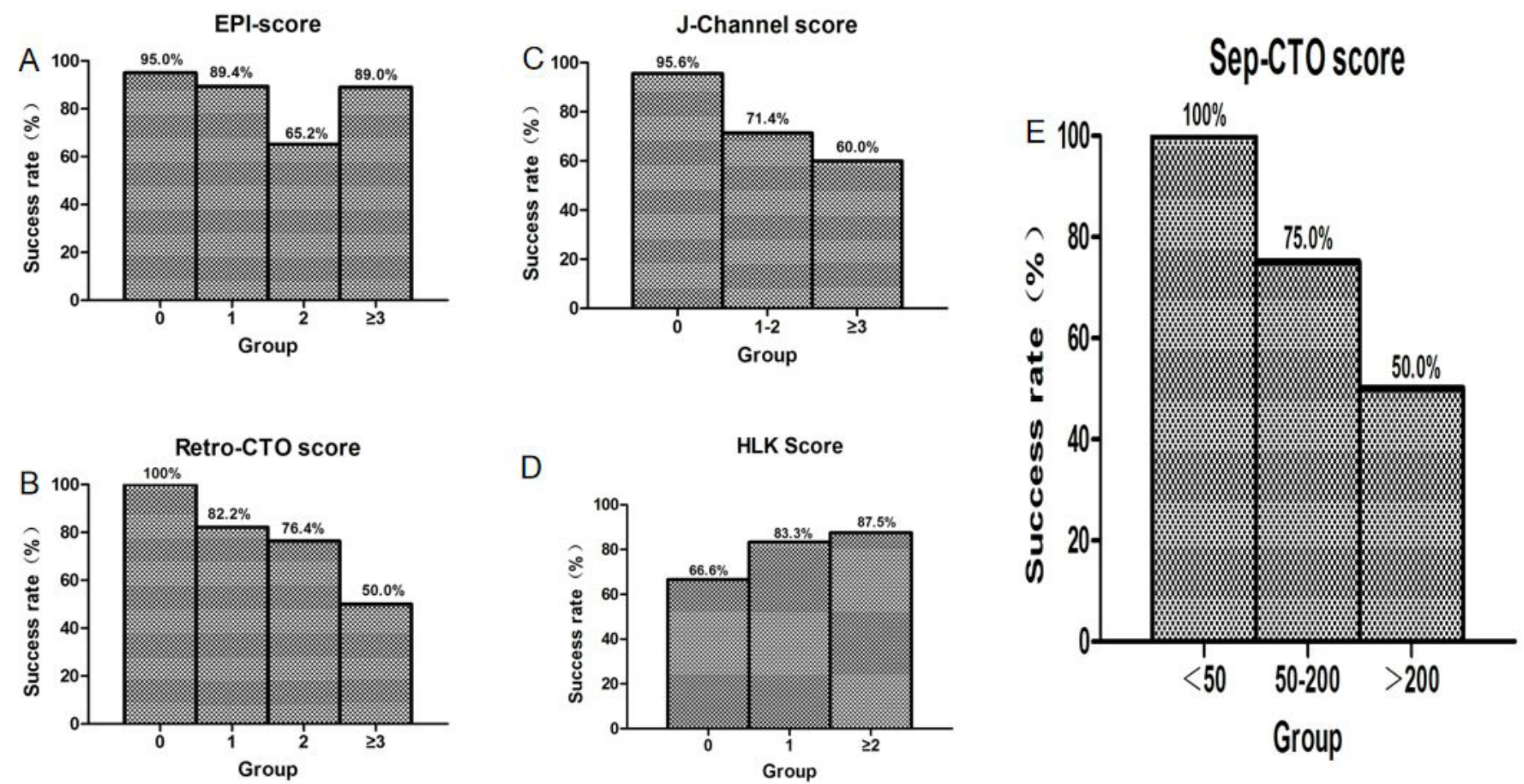

Figure 9

Probabilities of predicting success of guidewire crossing in different groups. EPI-score (A), Retro-CTO (B), J-Channel score (C), HLK score (D), and Sep-CTO score (E). 


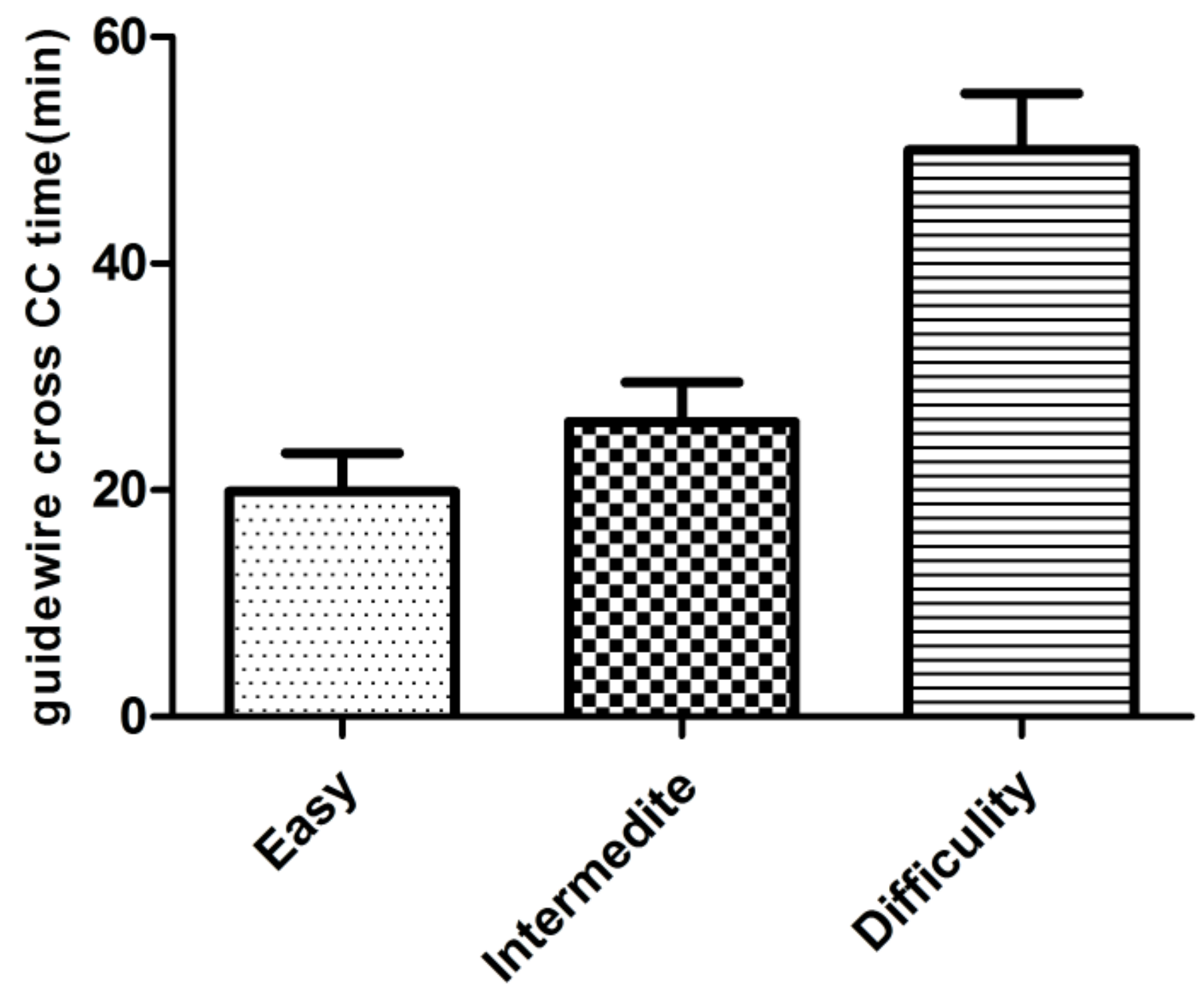

Figure 10

Time of GW crossing success using Sep-CTO score 


\section{Sep-CTO score}

A Diabetes

-Yes

-No

B Small size

-Yes

-No

C Crokscrew

-Yes

-No

D Side branch at tortuosity

-Yes

-No
Septal 68

98

100

80
Category of difficulty (total score)

-Easy: $<50 \quad$ (Risk: $<0.1$ )

-Intermediate:50-200 (Risk:0.1-0.5) -Difficult: $>\mathbf{2 0 0} \quad$ (Risk: $>0.5$ )
Total score

Figure 11

Summary of the Sep-CTO score 